\title{
Upper Airway Stimulation in the Management of Obstructive Sleep Apnea Syndrome: Neurostimulation of Hypoglossal Nerve
}

\author{
Peter Baptista ${ }^{1}$, Carlos Prieto ${ }^{2}$, Octavio Garaycochea ${ }^{3}$
}

\begin{abstract}
Obstructive sleep apnea (OSA) syndrome is one of the most prevalent chronic diseases in the general population. Continuous positive airway pressure (CPAP), is considered the gold standard therapy for its treatment, but adherence is a critical issue that decreases its use. Upper airway stimulation (UAS) through selective hypoglossal nerve stimulation has emerged as a non-anatomically modifying surgery has shown longterm improvements in objective respiratory and subjective quality-of-life outcome measures. It provides multilevel upper airway improvement through its action at the retrolingual and retropalatal portion of the airway, with the advantage of being an adjustable medical device. The two different commercially available implants are Imthera (LivaNova) and Inspire (Inspire Medical Systems). A large amount of information has been described mainly with the Inspire upper airway stimulation system. Patient selection criteria, implantation timeline, the operative procedure with activation and follow-up are discussed. Multiple studies that have shown significant improvements in both subjective and objective outcome measures as apnea hypopnea index (AHI), oxygen desaturation index (ODI), Epworth sleepiness scale (ESS), functional outcomes of sleep questionnaire (FOSQ) have been shown to be maintained respectively at long term follow-up.

Keywords: Continuous positive airway pressure, Functional outcomes of sleep questionnaire, Upper airway stimulation.

International Journal of Head and Neck Surgery (2019): 10.5005/jp-journals-10001-1381
\end{abstract}

\section{INTRODUCTION}

Obstructive sleep apnea (OSA) syndrome is one of the most prevalent chronic diseases in the general population and probably the most common respiratory disorder; recent data suggest that between $14 \%$ and $49 \%$ of middle-aged men have clinically significant OSA. ' However, due to the increase in the global obesity epidemic and its intimate association with OSA, the incidence and prevalence are expected to increase. ${ }^{2}$

It is estimated that between 15 and 19\% of the population has an apnea hypopnea index (AHI) greater than 10. This sleep disorder is associated with poor quality of life and health risk; nevertheless, an adequate diagnosis and a multidisciplinary treatment may provide improvement. $^{3}$

The main consequences of sleep apnea are the continuous oxygen desaturations and also metabolic, neurological, and cardiovascular disorders. Some signs and symptoms of obstructive sleep apneahypopnea syndrome (OSAHS) are obstructive apneas, hypopneas, or respiratory effort-related arousals, daytime symptoms, such as sleepiness, fatigue, or poor concentration and other signs as snoring, restlessness, or resuscitative snorts. In addition, severe untreated OSAHS is associated with increased all-cause and cardiovascular mortality. ${ }^{3}$

Even though the gold standard therapy for the treatment of OSAHS is continuous positive airway pressure (CPAP), multiple factors associated with this disease and the variable adherence to CPAP have driven the search for new surgical therapies in the last several years. ${ }^{4,5}$

One of the new surgical therapies is upper airway stimulation (UAS) through selective hypoglossal nerve stimulation. UAS is a nonanatomically modifying surgery first described in $2001^{6}$ and approved by FDA in 2014 with long-term improvements in objective respiratory and subjective quality-of-life outcome measures. ${ }^{7-9}$

UAS differs from traditional OSAHS surgery for sleep apnea in several key ways. One element critical to the success of UAS therapy
${ }^{1-3}$ Department of Otorhinolaryngology, Clínica Universidad Navarra, Pamplona, Navarra, Spain

Corresponding Author: Peter Baptista, Department of Otorhinolaryngology, Clínica Universidad Navarra, Pamplona, Navarra, Spain, Phone: +34637126088, e-mail: pmbaptista@unav.es

How to cite this article: Baptista P, Prieto C, Garaycochea O. Upper Airway Stimulation in the Management of Obstructive Sleep Apnea Syndrome: Neurostimulation of Hypoglossal Nerve. Int J Head Neck Surg 2019;10(4):77-85.

Source of support: Nil

Conflict of interest: None

is its ability to provide multilevel upper airway improvement with one procedure, not only the retro lingual space but also the retropalatal portion of the airway, at least in part due to mechanical coupling of the tongue to the palate and pharyngeal walls. UAS also has the advantage of being an adjustable medical device, which is important for a chronic long-term condition like OSAHS. The amplitude and other stimulation parameters can be titrated in the clinical or sleep laboratory setting to optimize both effectiveness and comfort across a longitudinal care model.

There are 2 different implants in the market at this moment, Inspire, Inspire Medical Systems ${ }^{\circledR}$ (Inc., Maple Grove, MN) and Imthera Inc., recently acquired by LivaNova a Cardiac and Neuromodulation Company. ${ }^{10}$ Both implants have shown very good results, but act in a slightly different manner.

The Imthera device stimulates the hypoglossal nerve at a more proximal location, co-activating the tongue protrusors and retractors to stiffen the posterior aspect of the tongue and pharyngeal walls to open the airway ${ }^{11}$ but when it is activated thanks to its 6 electrodes around the nerve, which allow selective activation of protruder fibers of the tongue. The Inspire system also stimulates the

() The Author(s). 2019 Open Access This article is distributed under the terms of the Creative Commons Attribution 4.0 International License (https://creativecommons. org/licenses/by-nc/4.0/), which permits unrestricted use, distribution, and non-commercial reproduction in any medium, provided you give appropriate credit to the original author(s) and the source, provide a link to the Creative Commons license, and indicate if changes were made. The Creative Commons Public Domain Dedication waiver (http://creativecommons.org/publicdomain/zero/1.0/) applies to the data made available in this article, unless otherwise stated. 
Table 1: Differences between Inspire and Imthera implants

\begin{tabular}{|c|c|c|}
\hline & Inspire & Imthera \\
\hline Implantable parts & - Stimulation lead with cuff & - Stimulation lead with cuff \\
\hline \multirow[t]{2}{*}{ Sensor lead } & - Breathing pressure sensor & - Implantable pulse generator \\
\hline & - Implantable pulse generator & \\
\hline Number of electrodes in cuff & 3 & 6 \\
\hline No. of incisions & 3 & 2 \\
\hline Tunneling & 2 & 1 \\
\hline Stimulation target & Hypoglossal nerve medial branch fibers (protruder) & Main branch \\
\hline Electrode type & Cuff electrodes & Cuff electrodes \\
\hline Power source & Implanted battery (life 10 years) & Rechargeable implanted battery (life 12 years) \\
\hline Stimulation pattern & "Phasic"/triggered & Tonic/"Continuous" \\
\hline
\end{tabular}

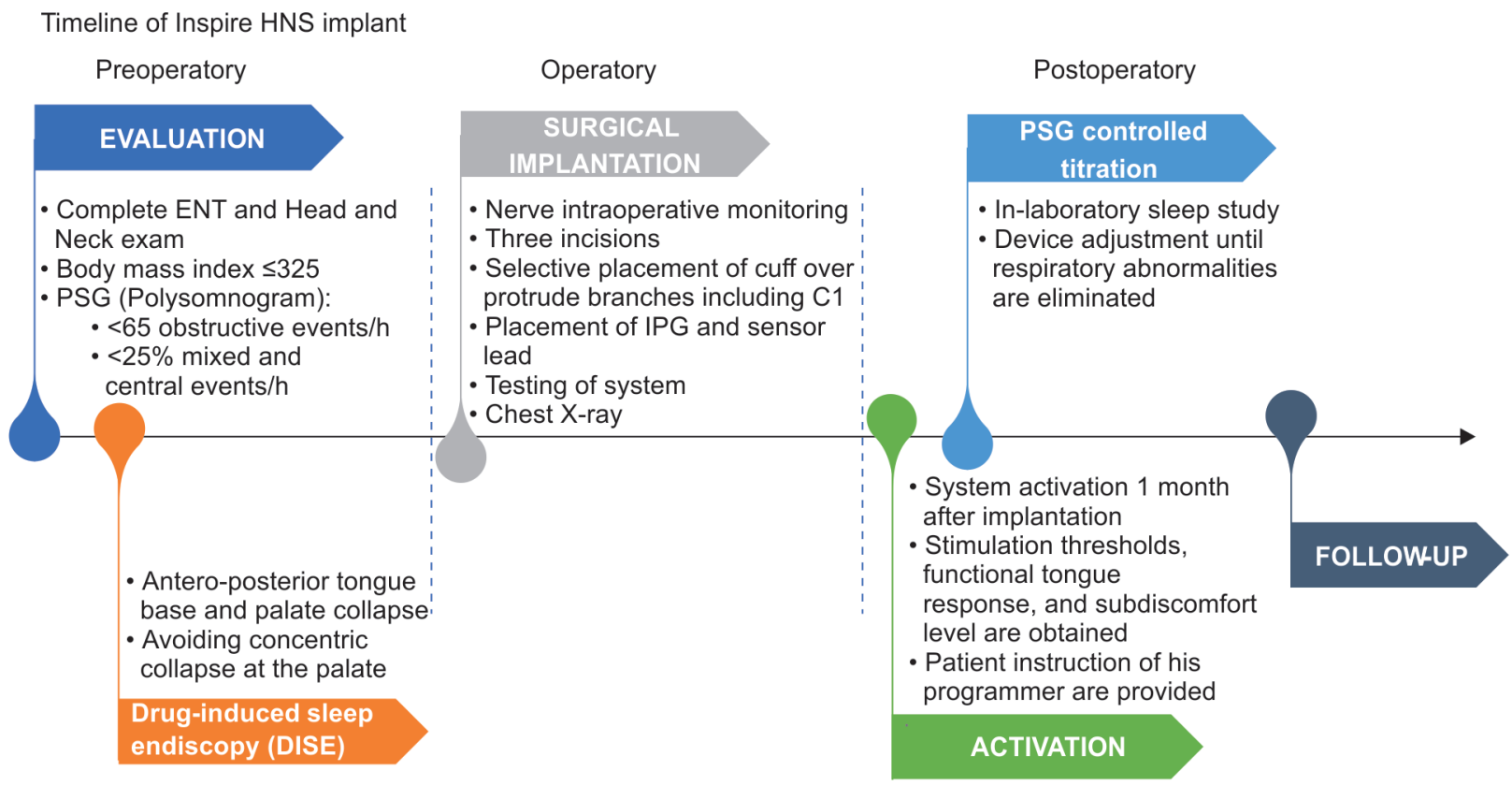

Fig. 1: Three different important stages for implantation

tongue, but only the genioglossus muscle fibers within placement of the stimulation lead directly over the ending nerve fibers of the hypoglossal nerve. The system also has a mechanism to sense respiration using a pulmonary pressure sensor, which Imthera does not have. The action of stimulation is slightly different, in Inspire it is "Phasic"/Triggered according to the patient's breathing, while Imthera is continuous. Please refer to Table 1 for differences.

The treatment we will refer to is the Inspire Medical Systems ${ }^{\circledR}$ (Inc., Maple Grove, MN) UAS therapy and involves functional electrical stimulation of the hypoglossal nerve as a means for increasing the posterior airway space at the level of the oropharynx and the hypopharynx.

UAS therapy has established itself as a promising new addition to the OSAHS treatment armamentarium. A recent multicenter prospective trial reported significant reduction in polysomnographic measures of disease severity ( $\mathrm{AHI}$ and oxygen saturation index) as well as in patient-reported quality of life measures that were maintained at 5 years follow-up, with overall low morbidity and good patient acceptance and adherence. ${ }^{9}$ The correct indication is essential to ensure therapeutic success. All patients implanted were suffering from moderate to severe
OSAHS according to the current FDA-approved guidelines for UAS therapy.

\section{Timeline}

The Inspire hypoglossal nerve stimulation timeline is set up in 3 different steps (Fig. 1):

- Patient selection

- Surgical procedure and immediate postoperative period within the first month after implantation

- Activation (1 month), titration and follow-up.

Each one of these steps is extremely important and implies the need for interdisciplinary collaboration. It is important to counsel the patient on device operation; the need for perioperative acclimatization, titration, and activation; and appropriate expectations of therapy.

\section{INDICATIONS OF UAS}

All patients being considered for UAS therapy must undergo a standard comprehensive sleep medicine evaluation and upper 
airway surgical consultation that includes an awake endoscopy and drug sleep induced endoscopy (DISE) which is mandatory.

UAS therapy is indicated for patients with the following:

- Patients older than 18 years

- Moderate to severe OSA with an AHI range of 15-65 per hour.

- Intolerance or inadequate adherence with CPAP:

- CPAP failure is defined as an inability to eliminate OSA (AHI of greater than 20 despite CPAP usage)

- CPAP intolerance is defined as inability to use CPAP (greater than 5 nights per week of usage; usage defined as greater than 4 hours of use per night)

- Unwillingness to use CPAP (for example, a patient returns the CPAP system after attempting to use it.

- $\mathrm{BMI}<32$

- Absence of complete concentric pattern of retropalatal collapse on DISE

- Less than $25 \%$ of central sleep apneas on a complete polysomnogram.

These inclusion criteria represent an over-simplification of the screening process. A complete evaluation of patient including history, comorbid sleep or medical disorders, craniofacial and upper airway anatomy, and other confounding factors must be sought. There are other patients' indication criteria as: Tonsil size smaller than type III or previously tonsillectomy, tongue free of malformations, symmetrical motor tongue activity, and absence of marked salivation alterations. There are changes in the criteria according to the type of device and the trading house.

\section{Contraindications}

- Obese patient with a BMI $>35$

- Central/mixed apneas $>25 \%$ of the total AHI

- Any anatomical finding that would compromise the performance of UAS, such as the presence of complete concentric collapse of the soft palate

- Preexisting conditions that have compromised neurological control of the upper airway

- Active psychiatric disease

- Comorbid nonrespiratory sleep disorders

- Patients who are unable or do not have the necessary assistance to operate the sleep remote control

- Patients who are pregnant or plan to become pregnant

- Patients who will require magnetic resonance imaging

- Patients with an implantable device that may be susceptible to unintended interaction with Inspire system, although they have descriptions of patients with simultaneous pacemaker, ${ }^{12}$ consult the device manufacturer to assess the possibility of interaction.

\section{Published Data on UAS Therapy with the Inspire ${ }^{\circledR}$ Upper Airway Stimulation}

Multiple studies have been performed since $2001,{ }^{6}$ but the most important one was published in 2014 as a multicenter trial called the stimulation therapy for apnea reduction (STAR) trial in the New England Journal of Medicine. ${ }^{13}$ In the STAR trial, eligible implant participants had moderate to severe OSA, CPAP intolerance, $\mathrm{BMI}$ of 32 or less, and absence of a complete circumferential pattern of palatal obstruction on DISE. After a rigorous clinical, polysomnographic, and DISE screening, 126 participants underwent surgical implantation of the HNS system and were followed for at least 12 months to assess the effectiveness and adverse events. Devices were titrated in the sleep laboratory during full-montage attended polysomnography, similar to CPAP titration, to optimize comfort and effectiveness. Primary outcome measures (AHI, 4\% ODI) and secondary outcomes measures (ESS, FOSQ) all demonstrated clinically and statistically significant improvements at 12 months (median AHI reduction from 29.3 to 9.0 and median ODI reduction from 25.4 to 7.4). Two-thirds of the implanted participants were considered successful responders to therapy by previously published surgical success criteria (median AHI 30 improvement to 6). Quality-of-life measures also improved significantly across the cohort with ESS reduced from median 11.0 to 6.0 and FOSQ increased from 14.6 to 18.2 at the 12-month follow-up.

Risk and morbidity data were very low, with no permanent hypoglossal nerve weakness, no serious device-related infection requiring explantation, and significantly less postoperative discomfort compared with traditional pharyngeal or skeletal sleep apnea surgeries. Adherence was excellent by self-report ( $86 \%$ of participants using the therapy nightly at the 12-month mark), but detailed objective data monitoring were limited.

At 12 months, a randomized therapy withdrawal study was performed in the first 46 responders, similar to prior withdrawal studies that have been performed using nasal CPAP. ${ }^{14}$ As with CPAP withdrawal studies, this study demonstrated that withdrawal of UAS therapy resulted in a recurrence of OSA severity, daytime sleepiness, and impaired quality of life.

Follow-up at 24 months reported clinically meaningful and statistically significant improvements in patient-centered OSA outcome measures, including snoring, daytime sleepiness, and sleep-related quality of life. The effect size on patient-centered outcome measures compared favorably with CPAP as well as other second-line therapies. ${ }^{15}$

These significant improvements in both subjective and objective outcome measures have been shown to be maintained respectively at 3,4, and 5 years of follow-up., ${ }^{9,14,17}$ A total of 97 participants (78\%) completed the scheduled 60-month clinical follow-up, and 71 participants (78\%) completed a voluntary 60 -month PSG. AHI response rate (AHI $\backslash 20$ events per hour and $50 \%$ reduction) was $75 \%(n=71)$. Long-term bed partner-reported and self-reported snoring reports demonstrated improvement from baseline and remained relatively stable from 12 to 60 months. Serious device-related events all related to lead/device adjustments were reported in $6 \%$ of the patients. ${ }^{9}$

Heiser et al. ${ }^{18}$ have reported their experience in UAS in a single German hospital. In 31 patients who received a UAS device (Inspire Medical Systems), mean preimplantation AHI of 32.9/hour could be reduced to $7.1 /$ hour after 12 months, and mean preimplantation ESS of 12.6 could be reduced to 5.9 after 12 months. Serious adverse events did not occur. Therapy adherence was a usage of 6.6 hour/ night after 12 months.

Heinser's team have also published their results ${ }^{19}$ in a multicenter prospective single-arm study under a common implant and follow-up protocol in three German centers including 60 patients. Every subject reported improvement in sleep and daytime symptoms. The average usage time of the system was $42.9 \pm 11.9$ hour/week. The median apnea-hypopnea index was significantly reduced at 6 months from 28.6/hour to 8.3/hour. No patient required surgical revision of the implanted system. 
At Pittsburgh, Kent et al. ${ }^{20}$ have reported their experience after the first 20 cases. Mean AHI (33.3 $\pm 13.0-5.1 \pm 4.3)$ and mean ESS (10.3 $\pm 5.2-6.0 \pm 4.4)$ decreased significantly. Seventy percent (14/20) of patients achieved a treatment AHI <5, 85\% (17/20) an AHI $<10$, and $95 \%(19 / 20)$ an $\mathrm{AHI}<15$. Adherence monitoring via device interrogation showed high rates of voluntary device use (mean $7.0 \pm$ 2.2 hour/night). We have also informed of our first experience in Spain with the device with a great improvement in AHI, ODI, and ESS. ${ }^{21}$

Also, Steffen et al. ${ }^{22}$ published the results of 60 participants, in which the median $\mathrm{AHI}$ reduced from 28.6 to 9.5 (baseline to 12 months). Patient-reported outcome measured in ESS and FOSQ both improved significantly from baseline to 12 months. The average usage time was $39.1 \pm 14.9$ hours per week among all participants based on the recordings by the implanted device.

A registry study has been opened across multiple institutions in the United States and Germany. To date, it represents the largest retrospective and prospective patient cohort study with this therapy. ${ }^{23}$ Recent results have shown in 301 patients enrolled between October 2016 and September 2017, with an improvement in $\mathrm{AHI}$, that decreased from $35.6 \pm 15.3$ to $10.2 \pm 12.9$ events per hour $(p<0.0001)$, and ESS scores that decreased from $11.9 \pm 5.5$ to $7.5 \pm 4.7$ ( $p<0.0001)$ from baseline to the post-titration visit. Patients utilized therapy for 6.5 hours per night. There were low rates of procedure- and device-related complications. Clinical global impression scores demonstrated that the majority of physicians (94\%) saw improvement in their patients' symptoms with therapy. The majority of patients (90\%) were more satisfied with UAS than CPAP. Adherence reported is similar to that described in the STAR trial and also in German postmarket outcomes. ${ }^{9,16,17}$

Shah et al. ${ }^{24}$ have performed a retrospective study comparing UPPP results with UAS, which shows better results with hypoglossal nerve stimulation in outcome measures for select patients with moderate to severe OSA with inability to tolerate CPAP.

Furthermore, Pietzsch et al., ${ }^{25}$ using a model to predict cardiovascular endpoints (myocardial infarction (MI), stroke, hypertension), motor vehicle collisions, mortality, quality-adjusted life years (QALYs), and costs, have shown that UAS substantially reduced event probabilities over 10 years (relative risks: MI 0.63; stroke 0.75; MVC 0.34) and was projected to add 1.09 QALYs over the patient's lifetime. Costs were estimated to increase by $\$ 42,953$, resulting in a lifetime incremental cost-effectiveness ratio of $\$ 39,471 /$ QALY. This study clearly indicates that UAS is a cost-effective therapy in the U.S. healthcare system.

Thus, UAS is currently playing a major role in treatment of OSAHS patients and can be implemented successfully into routine clinical practice, outside of a trial setting. As any surgical procedure, there is a learning curve to perform UAS; ${ }^{26}$ Murphey et al. ${ }^{27}$ have reported that, during STAR trial, surgical time for implantation of the UAS system decreased significantly after the first five implants and then stabilized.

\section{System Components of Inspire ${ }^{\circledR}$ UPPer Airway Stimulation}

The Inspire ${ }^{\circledR}$ UAS system is composed of a stimulation lead, a sensing lead, and an implantable pulse generator (IPG), which together sense respiration patterns and delivers stimulation to the hypoglossal nerve synchronously with inspiration (Fig. 2).

\section{Sensing Lead}

The sensing lead incorporates a differential pressure sensor that detects respiratory cycles by their pressure variations. The pressure waveform is monitored by the IPG, which triggers stimulation therapy synchronously with respiration, and is placed between the inner and outer intercostal muscles at the level of the 5th or 6th right intercostal space.

\section{Stimulation Lead}

The stimulation lead incorporates a cuff section that includes 3 electrodes that can be arranged in a variety of unipolar or bipolar electrode configurations for stimulation, placed at the level of the protruding fibers of the right hypoglossal nerve.

\section{Implantable Pulse Generator}

The IPG contains an algorithm that synchronizes hypoglossal nerve stimulation with respiration signals. The algorithm parameters can be adjusted as per the needs of each patient. The IPG's electronics and battery are sealed inside a titanium case. The connector module on top of the IPG attaches to the sensing and stimulation leads. This device is placed in a pocket at the right infraclavicular region.

\section{Physician Programmer}

The physician programmer consists of a tablet computer and a telemetry unit. The telemetry unit connects with the IPG through clothing/skin via short-range radiofrequency telemetry. It allows the physician to noninvasively examine the IPG status (e.g., battery status and UAS system patient usage), adjust stimulation and sensing parameters, monitor respiratory waveforms, and store waveforms and patient programmed settings. The telemetry unit is powered by a wall outlet connection and wirelessly communicates via Bluetooth with the physician programmer tablet.

\section{Patient Programmer}

The patient programmer is an external device about the size of a smartphone that is used by the patient to activate nerve stimulation before sleep. The patient places the programmer over his or her body at the implanted IPG site and uses the buttons on the programmer to turn the therapy on or off, temporarily suspend therapy, or make adjustments to the stimulation amplitude (withinphysician preselected limits).

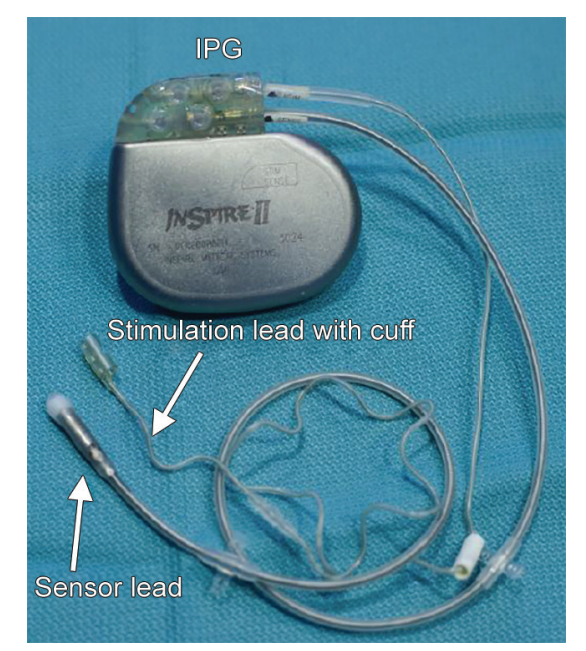

Fig. 2: Components of the implant that go inside the body 


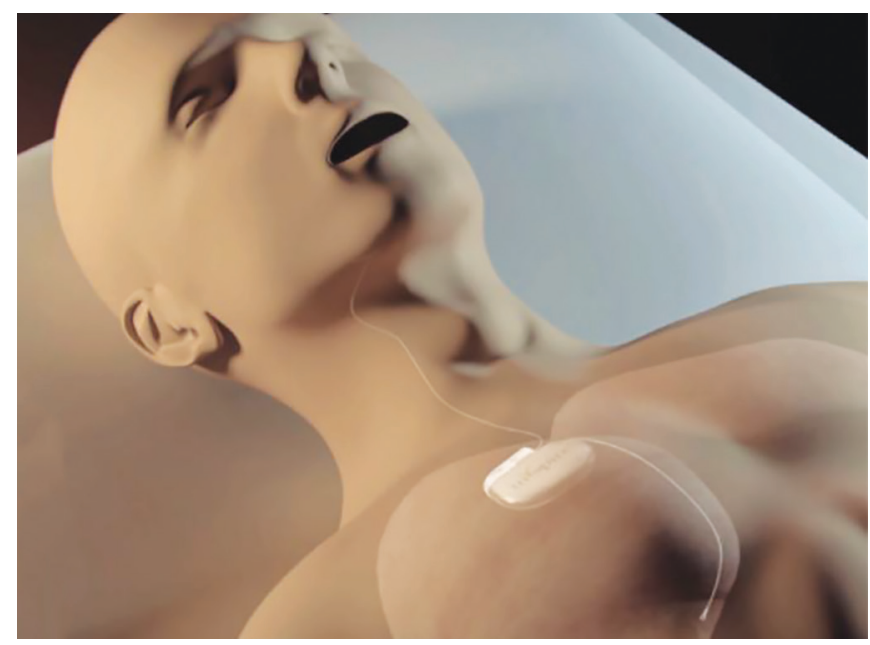

Fig. 3: Animation of the implant

\section{Operative Technique of Inspire ${ }^{\circledR}$ Upper Airway Stimulation}

Conceptually, the procedure may be divided into nine parts: ${ }^{28-31}(1)$ preparation of incision sites, ${ }^{23,24}$ (2) placement of stimulation lead, (3) verification of tongue response to stimulation, (4) placement of IPG, (5) tunneling of stimulation lead to IPG, (6) placement of sensing lead, (7) tunneling of sensing lead to IPG, (8) verification of sensing, and (9) closure of incisions. Precise order of the major steps can be expected to vary within reason, according to the preferences of the surgeon and the unique conditions of a particular implant procedure (Fig. 3).

\section{Operating Room Preparations}

The UAS system is implanted under general anesthesia through three surgical incisions in the neck and chest area made on the patient's right side. Choosing the right side improves pressure signals of the sensing lead owing to reduced cardiac pressure oscillations compared with the left side. But there are circumstances that could make necessary for placement on the left side as hobbies (hunting, etc.), previous surgical operations, etc. ${ }^{30}$

EMG bipolar electrode needles are placed in the lateral aspect of the tongue and in the floor of the mouth, these electrodes are connected to an intraoperative nerve monitoring system. Inserting bipolar EMG electrodes ${ }^{28}$ toward the mid-inferior section of the tongue targets the genioglossus muscle. The styloglossus muscle may be targeted for definitive exclusion by inserting bipolar EMG electrodes along the ventrolateral surface of the tongue. ${ }^{29}$ Both electrodes are connected to the nerve integrity monitoring system.

Adequate preparation measures (i.e., fastidious sterile preparation) should be taken to minimize the risk of device-related infection similar to the surgical procedures for an electrically active implanted system. ${ }^{28}$ The neck is slightly extended and the right arm is positioned to allow better visualization of the chest area where the respiration sensor is to be placed. Prophylactic antibiotics (e.g., amoxicillin + clavulanic acid) are given intravenously at the onset of anesthesia. Long-acting muscle relaxants must be avoided to accommodate peri- and intraoperative examination of tongue motion in response to electrical stimulation. Transnasal intubation is recommended for the same purpose. Furthermore, a gauze packing or a bite block of appropriate thickness is placed between

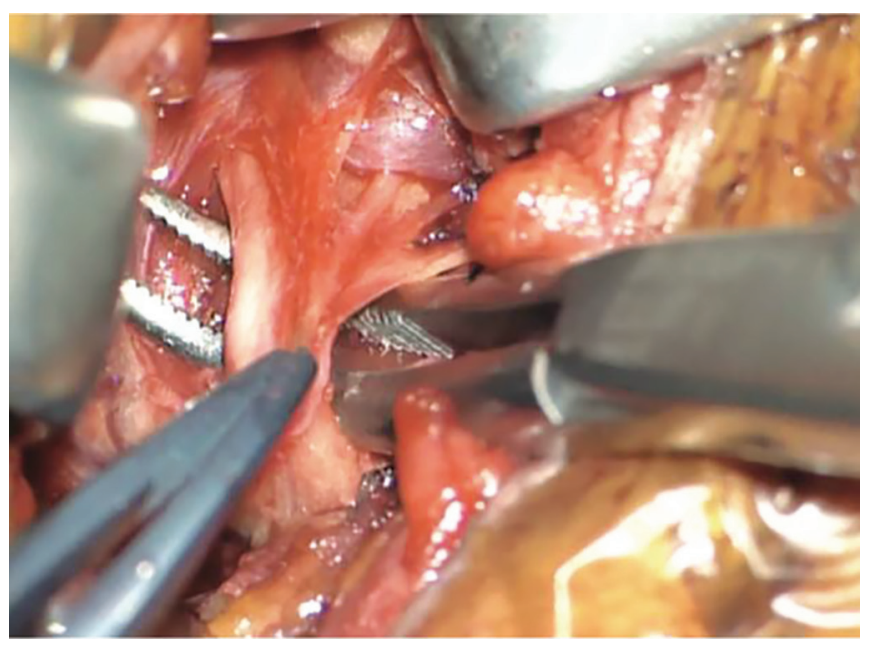

Fig. 4: A meticulous dissection of the hypoglossal nerve, searching for the protruder fibers (genioglossus) and excluding the retruder fibers (hyoglossus and styloglossus)

the molar teeth of the left side, to facilitate the inspection of tongue movement during intraoperative assessment of tongue response. The activity of the tongue muscle fibers is monitored using a nerve integrity monitoring system. The surgeon should also visually monitor tongue movement through a transparent draping over the patient's mouth.

\section{Placement of Stimulation Lead}

The first step of the procedure is the identification of the hypoglossal nerve and placement of the stimulation electrode to confirm a positive tongue response to stimulation (Fig. 4). A horizontal incision $3 \mathrm{~cm}$ inferior to the inferior border of the mandible is performed, to avoid injury to the marginal mandibular branch of the facial nerve. From the anterior portion of the submandibular gland for a length of approximately $4-6 \mathrm{~cm}$ long, depending on the neck, over a cervical relaxed skin tension line. The platysma is incised and the inferior border of the submandibular gland is identified and followed down to the intermediate tendon of the digastric muscle, which runs superior to the greater cornu of the hyoid bone.

The hypoglossal nerve main trunk can be identified in this location, at the anterior edge of the submandibular gland. The large ranine vein (vena comitans of hypoglossal nerve) most often overlies the anterior branching of the hypoglossal nerve, may be suture ligated or gently separated to avoid damaging the nerve, although it has been recently said that ligation of the concomitant vena comitans (aka, ranine vein) during implantation and cuff positioning can create damage to the nerve and its supporting structures. This may result in temporary tongue weakness, a sign of mild postoperative neurapraxia. ${ }^{32}$

Bipolar electrical cautery should be used throughout the procedure, with special care near the nerve and implanted components.

Once this has been accomplished, the anterior branching is investigated, isolating the retraction branches to be excluded (HG and SG) and the protrusion branches to be included (GG). The nerve integrity monitor is crucial to this identification. It is necessary to use the nerve integrity monitoring system (NIM) to verify that all exclusion and inclusion fibers for cuff placement. Once the protruder fibers are isolated, the cuff of the stimulator electrode is placed around the nerve branches to be included. The objective is to achieve an "unhindered protrusion of the stiffened tongue," 
in which unhindered implies to exclude all extrinsic retractors (hyoglossus and styloglossus), protrusion to include all extrinsic protrusors (oblique and horizontal genioglossi), and stiffened to include all intrinsic stiffeners (transversal and vertical). ${ }^{33}$ It is recommended to include the first cervical nerve (C1), innervating the geniohyoid muscle, which seems to be important for the opening at the level of the hyoid. ${ }^{34}$ One of the main key factors during implantation is to determine the last exclusion branch of the hyoglossus muscle to ensure comprehensive exclusion of tongue retractors.

The stimulation lead is then brought under the digastric muscle, secured, and the rest of the lead is guided under the platysma muscle toward the subclavicular area to connect with the IPG.

\section{Placement of IPG and Tunneling of Stimulation Lead}

The IPG is typically implanted in a subcutaneous pocket in the right mid infraclavicular region, superficial to the pectoralis major muscle fascia to accommodate the IPG. The stimulation lead is subcutaneously tunneled into the pocket space with a tunneling device or a long Kelly clamp. The lead should be kept in a subplatysmal plane for cosmetic reasons.

\section{Placement and Tunneling of Sensing Lead}

A third incision approximately $5 \mathrm{~cm}$ long is made horizontally at the fifth to sixth right intercostal space, with the lateral edge anterior to the mid-axillary line. The dissection is carried down to the posterior border of the pectoralis major muscle for access to intercostal muscles. Ribs should be palpated to identify the intercostal space trajectory. The paired serratus anterior and external intercostal muscles should be carefully spread, layer by layer.

Once identification of the internal intercostal muscle is achieved by its fibers running in an oblique manner opposite to the external intercostal muscles, a tunnel of $3-5 \mathrm{~cm}$ is created using a malleable valve curved to desire length between the both intercostal muscles. The respiration sensor is placed within this tunnel, with the sensing side facing the lung.

Care should be taken to avoid dissecting infracostally, as the intercostal neurovascular bundle is located at the inferior border of the rib. The lead is fixed by suturing the anchors of the cable to the tissues, in an omega type fashion allowing the natural movement of the area without the creation of tension between the sensing lead and the IPG. The sensing lead is then tunneled subcutaneously toward to the IPG pocket space using the tunneling device or long Kelly clamps and then connected to the IPG.

\section{Testing of Sensing and Closure of Incisions}

Once the three components are implanted, the testing of the system follows. The physician programmer module is used to verify and store respiration signals. The function of the entire system (respiration sensor, IPG, and stimulation lead) at this point could be verified, if deemed appropriate, by the surgical team. First, the pleural respiratory sensing waveform is assessed, to ensure good function of the sensor ${ }^{30}$ and after stimulation of the tongue is initiated at the standard electrode configuration gradually adjusting stimulation in small increments, ensuring adequate tongue mobility, not only visually but also in the EMG tracing of the nerve monitoring system. If there might be any problem, a meticulous review of each previous step must be considered.

The size of the IPG pocket is matched as closely as possible to the IPG dimensions so the IPG and extra length of stimulation and sensing leads wrapped under the IPG t snugly with minimal movement. The IPG is sutured in a hanging position to the underlying fascia, with the IPG's laser-inscribed surface facing out. At the same time, minimal movements of the IPG owing to shoulder and chest movements remain possible. Meticulous homeostasis is achieved, and all wounds are copiously irrigated.

All wounds are closed in multiple layers, with fastidious attention to proper coverage of all internal prosthetics. In general, drainage is not recommended to reduce the potential for infectious contamination of the implanted components. Pressure dressings should be applied, although they are not necessary in all cases.

\section{Postoperative Care}

The patient stays overnight in the hospital. An anteroposterior chest $X$-ray and lateral neck X-ray are obtained to document the position of the IPG and the leads and to rule out an ipsilateral pneumothorax. Ideally, the chest X-ray incorporates the upper neck as well as the lung fields to allow visualization of the entire implant. Because this is not standard for X-ray technicians, special instructions are necessary to facilitate the appropriate images. Also the X-ray may prove useful in case of future malfunctioning or accident in order to see if displacement of electrodes has occurred (Fig. 5)

Common pain killers (e.g., paracetamol) are sufficient in most cases and may be used during postoperative days $2-5$. Patients should avoid vigorous movement of the right arm and shoulder for a period of at least 3-4 weeks. Wound care instructions are provided, with a follow-up arranged within 10 days for evaluation of all wounds and suture removal. Patients are explicitly advised that the device remains inactive and should not expect to feel any stimulation. Vigorous activities can be resumed 1 month after implantation, with appropriate protection of the IPG and leads.

\section{Morbidity}

As the procedure does not open a major body cavity or viscera, morbidity is low. Pain is mostly related to the incisions and until now has not lasted more than a few days. Hematoma and seroma can occur, eventually requiring antibiotic or surgical treatment.

If any component becomes contaminated and causes repetitive or chronic infection that is resistant to common therapy, the system should be explanted. Temporary tongue weakness may occur during the first 2-3 weeks after the implant. Hypoglossal palsy has not been described.

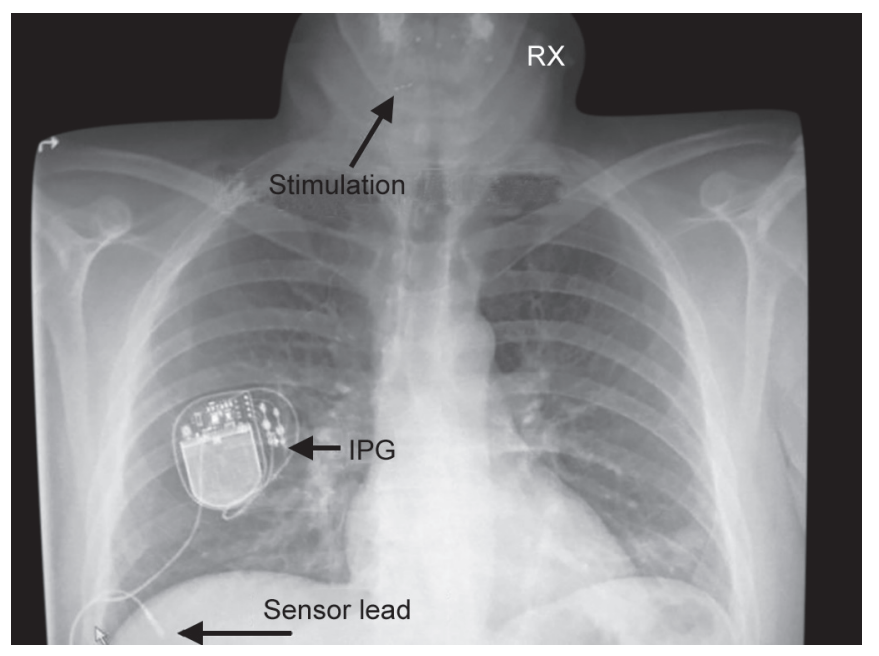

Fig. 5: Postoperative day 1 chest X-ray. The implanted components can be seen. It is useful to have as baseline evidence 


\section{Special Considerations of Surgery in Women}

Although technique for implantation of UAS is similar in men and women, there are some special concerns regarding the procedure in women. A previous history of previous breast surgery, particularly reconstructive surgery or breast augmentation, is important. The surgeon should review operative reports to aid in preoperative planning for UAS implantation. Additionally, history should be obtained regarding a personal or family history of breast cancer; although magnetic resonance imaging (MRI) is not routinely used for breast cancer screening, MRI may become an important imaging modality for patients at high risk for breast cancer. Such patients should be counseled regarding obtaining an MRI prior to UAS implantation given that MRI is contraindicated for now after placement of the current UAS device. ${ }^{35}$ Finally, it is helpful to ask patients to outline the position of their bra strap and its underwire immediately prior to the procedure so that incisions can be appropriately positioned intraoperatively.

Women may have special concerns regarding the location of two separate incisions required for placement of the UAS device. For cosmetic reasons, the upper chest incision may be placed slightly lower than normal with the incision over the implant. The lower placement of the incision will allow the IPG to be buried in the upper portions of breast tissue and somewhat mask the bulge that can be associated with the IPG. Also, the incision should be positioned medial to the location of their bra strap to avoid discomfort that may occur if the strap were to ride over the IPG. The lower chest incision should be positioned lower than the location of the bra underwire in order to avoid surgical scar irritation and discomfort. ${ }^{30}$

Mammography is not contraindicated postimplantation. Patients should continue to receive routine mammography, as per current guidelines.

\section{System Activation and Titration}

According to the timeline the UAS system is activated 1 month after the procedure, and stimulation amplitude thresholds for nerve capture, functional tongue response, and subdiscomfort level are obtained during wakefulness, these are done by the neurophysiologists in our hospital center (Fig. 6). At the same time, the patient is instructed about his programmer (remote control) and taught how he/she can adjust the level of intensity, turn on, pause, and turn off the device. The patient may adjust the stimulation

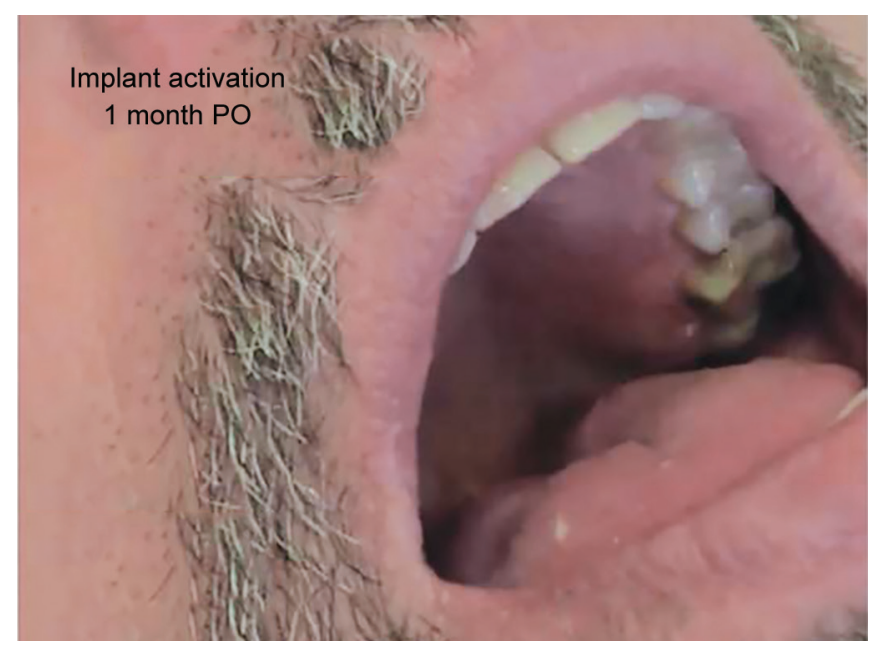

Fig. 6: Tongue movement during activation voltage within a certain range predefined by the physician according to therapy titration during sleep before going to bed.

During this period, four different tongue motions can be seen after implantation: right protrusion (RP), bilateral protrusion (BP), left protrusion (LP), and mixed activation (MA). RP shows a protrusion of the tongue over the lower teeth with deviation to the left side. BP shows a clear protrusion of the tongue over the lower teeth without deviation to one side. These two phenotypes were correlated with better therapy response. MA includes every other kind of tongue motion such as shortening or furling of the tongue. ${ }^{32,33}$

Tongue motion patterns such as left protrusion (LP) of the tongue (i.e., contralateral extension of the tongue) and mixed activation (MA) (i.e., includes every other kind of tongue motion such as shortening or curling of the tongue) were associated with less favorable results.

BP and LP may be caused by crossing of the nerve plexus of the hypoglossal nerve to genioglossus muscle in the tongue. There is an individual variability in branching of the nerve endings as shown by Mu and Sanders ${ }^{36}$ in human cadaver, which may explain the different postoperative tongue motions. Kubin et al. ${ }^{37}$ showed in 2015 that $50 \%$ patients have a cross-linked motor innervation of the hypoglossal nerve.

BP and RP are probably common with a distal cuff placement at the hypoglossal nerve with almost activation of the horizontal and oblique genioglossus muscle fibers. In most of the cases, the soft palate can open freely. This could be related to the activation of the palatoglossus muscle in which tongue motion brings the palatoglossal muscle also forward.

MA consists of any other kind of motion or protrusion of the tongue, such as a retrusion or shortening of the tongue. This occurrence is related to a more proximal placement of the cuff. Nevertheless, it may lead to a fully open tongue base but with different variations on the soft palate (obstruction or opening). Safiruddin et al. ${ }^{38}$ showed that both responders and nonresponders to the UA stimulation therapy had similar degrees of retro lingual opening to stimulation, but responders had a greater increase in the retropalatal area.

Heiser et al. have informed that patients with a bilateral protrusion show a more significant opening at the retropalatal level compared to ipsilateral protrusion. ${ }^{39}$ Follow-up visits are necessary during the first year to adjust the settings of the patient programmer, as the patient grows more accustomed during the first months of treatment.

\section{Titration with a Polysomnography}

This is an in-laboratory sleep study and involves device adjustment until respiratory abnormalities are eliminated. It is performed with a programming device, which modifies settings on the device to optimize control of OSA and patient comfort. The parameters that are identified as best for the patient are then utilized, within a narrow range, to set the patient's device. Patients are instructed to use their remote control to increase or decrease voltage, within this range, to mitigate discomfort and maximize positive effects on sleep and snoring.

Advanced titration of UAS therapy involves changes of the electrode configurations to optimize muscle recruitment, which can, in some instances, convert an initial nonresponder into a responder. ${ }^{33,34}$ Different types of electrode configurations may result in different tongue motion, variable palate movement 


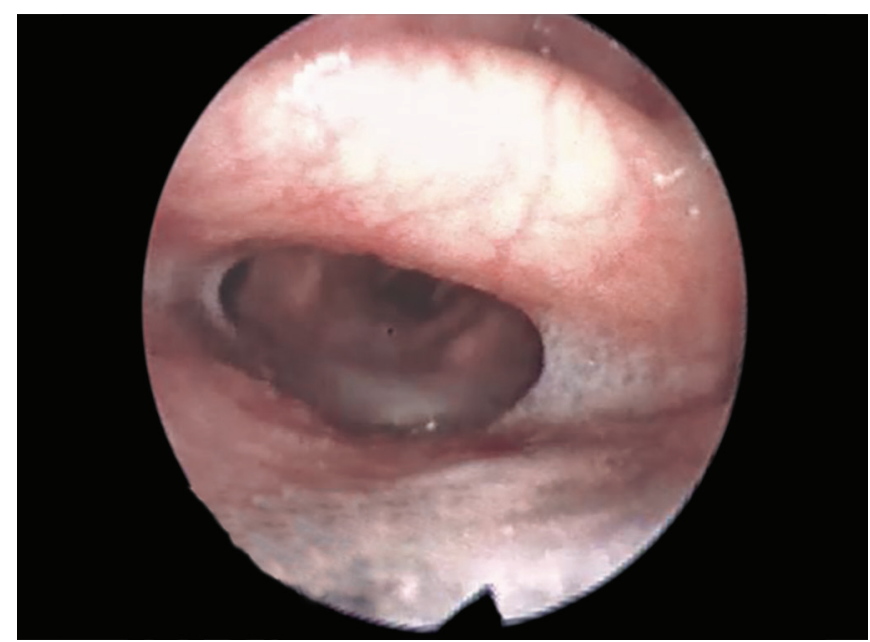

Fig. 7: Drug-induced sleep endoscopy (DISE) of implanted patient. Opening of the airway is seen at the level of the tongue base and palate

with diverse therapy outcomes. To this end, it is important to understand the mechanism of tongue muscle activation resulting from hypoglossal nerve stimulation under various electrode configurations. The closed-loop UAS system employed offers five electrode configurations that create different electric fields and effects on branches of the hypoglossal nerve. There are various possible mechanisms that explain changes of tongue motion with different stimulation voltages and different electrode configurations. Tongue movement patterns are likely affected by the type of nerve branches included in the cuff, electric field, voltage, and cuff rotation. During UAS device programming, in addition to voltage adjustment, electric field setting can be changed as necessary to optimize the therapy response.

It may be necessary to periodically perform titration at least once a year after the first titration depending upon the patient response, the previous sleep study, and also previously reached parameters.

On occasions, it is useful to perform a DISE post-titration in order to observe tongue movement (Fig. 7).

\section{New Considerations}

Recently, Diercks et al. ${ }^{40}$ published a series of 6 pediatric patients with Down syndrome that were implanted with hypoglossal nerve stimulator. After appropriate device titration, the nerve stimulator was effective in relieving upper airway obstruction with a greater than $50 \%$ reduction in $\mathrm{AHI}$ in all patients. In patients reliant on tracheotomy and CPAP, nerve stimulator therapy was so successful that these therapies could be discontinued after initial titration sessions. Patients not only exhibited an improvement in their $\mathrm{AHI}$ but also showed clinically significant improvement in their QoL based on validated QoL instruments. As time passes, there is a better knowledge of pathophysiology of OSA and new indications will probably be suggested for use of the UAS. Improvements in anatomical knowledge, electrode lead IPG size, types of stimulus, and sensor lead will allow an improvement of results in a greater number of patients.

\section{CONCLUSION}

UAS is a novel therapy for treatment of the moderate to OSA in selected patients. Surgery of hypoglossal nerve stimulation requires certain knowledge of the anatomy of the submental region; and even though neck anatomy is typically straightforward and predictable, anatomy variations or loss of the dissection plane in the surgery can mislead us. To improve UAS therapy outcomes, in addition to careful candidate selection during DISE, intraoperative nerve identification and exclusion of tongue retractor nerve branches is essential and can be best achieved by nerve monitoring. However, despite the nerve monitoring, the resulting tongue motion at activation and beyond can be variable. Tongue motion pattern can make a difference in therapy outcomes and requires meticulous assessment. Changes in electrode configuration can result in tongue motion changes.

An adequate patient selection is necessary to reach good results. There are many questions that will be answered in the next years, and improvements to be made in the implant (size, biocompatibility with other electromagnetic systems, improvement of breathing sensor and also in the cuff placement). Additional features can be added such as body position adjustment, especially for patients that suffer from positional OSA. With time, all these questions may be answered.

\section{References}

1. Garvey JF, Pengo MF, Drakatos P, et al. Epidemiological aspects of obstructive sleep apnea. J Thorac Dis 2015;7(5):920-929.

2. Punjabi NM. The epidemiology of adult obstructive sleep apnea. Proc Am Thorac Soc 2008;5:136-143. DOI: 10.1513/pats.200709-155MG.

3. Plaza G, Baptista P, O'Connor C. Diagnóstico y tratamiento de los trastornos respiratorios del sueño, 1st ed., España: Autor-Editor; 2015.

4. Weaver TE, Grunstein RR. Adherence to continuous positive airway pressure therapy. The challenge to effective treatment. Proc Am Thorac Soc 2008;5:173-178. DOI: 10.1513/pats.200708-119MG.

5. Rotenberg BW, Murariu D, Pang KP. Trends in CPAP adherence over twenty years of data collection: a flattened curve. J Otolaryngol Head Neck Surg 2016 Aug 19;45(1):43. DOI: 10.1186/s40463-016-0156-0.

6. Schwartz AR, Bennett ML, Smith PL, et al. Therapeutic electrical stimula-tion of the hypoglossal nerve in obstructive sleep apnea. Arch Otolaryn-gol Head Neck Surg 2001;127:1216-1223. DOI: 10.1001/ archotol.127.10.1216.

7. Heiser C, Thaler E, Boon M, et al. Updates of operative techniques for upper airway stimulation. The Laryngoscope 2016;126:S12-S16. DOI: 10.1002/lary.26158.

8. Woodson B, Soose R, Gillespie M, et al. Three-Year Outcomes of Cranial Nerve Stimulation for Obstructive Sleep Apnea: The STAR Trial. Otolaryngology-Head and Neck Surg 2015;154(1):181-188. DOI: $10.1177 / 0194599815616618$.

9. Woodson BT, Strohl KP, Soose RJ, et al. Upper Airway Stimulation for Obstructive Sleep Apnea: 5-Year Outcomes. Otolaryngology-Head and Neck Surg, https://doi.org/10.1177/0194599818762383.

10. Wray CM, Thaler ER. Hypoglossal nerve stimulation for obstructive sleep apnea: A review of the literature. World J Otorhinolaryngol Head Neck Surg 2016 Dec 22;2(4):230-233. DOI: 10.1016/j.wjorl.2016.11.005.

11. Zaidi FN, Meadows $\mathrm{P}$, Jacobowitz $\mathrm{O}$, et al. Tongue anatomy and physiology, the scientific basis for a novel targeted neurostimulation system designed for the treatment of obstructive sleep apnea. Neuromodulation 2013; Aug;16(4):376-386. DOI: 10.1111/j.15251403.2012.00514.x.

12. Parikh V, Thaler E, Kato M, et al. Early feasibility of Hypoglossal Nerve Upper Airway Stimulator in Patients with Cardiac Implantable Electronic Devices and Continuous Positive Airway PressureIntolerant Severe Obstructive Sleep Apnea. Heart Rhythm 2018 Apr 17;15, 8, 1165-1170. DOI: 10.1016/j.hrthm.2018.04.016.

13. Strollo PJ Jr, Soose RJ, Maurer JT, et al. STAR trial group. Upper airway stimulation for obstructive sleep apnea. N Engl J Med 2014;370: 139-149. DOI: 10.1056/NEJMoa1308659. 
14. Woodson BT, Gillespie MB, Soose RJ, et al. STAR Trial Investigators. Randomized controlled withdrawal study of upper airway stimulation on OSA: short- and long-term effect. Otolaryngol Head Neck Surg 2014;151:880-887. DOI: 10.1177/0194599814544445.

15. Soose RJ, Woodson BT, Gillespie MB, et al. STAR trial Investigators. Upper airway stimulation for obstructive sleep apnea: self-reported outcomes at 24 months. J Clin Sleep Med 2016;12:43-48. DOI: $10.5664 /$ jcsm. 5390 .

16. Woodson BT, Soose RJ, Gillespie MB, et al. STAR Trial Investigators. Three-year outcomes of cranial nerve stimulation for obstructive sleep apnea: the STAR trial. Otolaryngol Head Neck Surgery 2016;154:181-188. DOI: 10.1177/0194599815616618.

17. Gillespie MB, Soose RJ, Woodson BT, et al. STAR Trial Investigators. Upper Airway Stimulation for Obstructive Sleep Apnea: PatientReported Outcomes after 48 Months of Follow-Up. Otolaryngol Head Neck Surg 2017;156(4):765-771. DOI: 10.1177/0194599817691491.

18. Heiser C, Knopf A, Bas M, et al. Selective upper airway stimulation for obstructive sleep apnea: a single center clinical experience. Eur Arch Otorhinolaryngol 2017 Mar;274(3):1727-1734. DOI: 10.1007/s00405016-4297-6.

19. Heiser C, Maurer JT, Hofauer B, et al. Outcomes of upper airway stimulation for obstructive sleep apnea in a multicenter German postmarket study. Otolaryngol Head Neck Surg 2017 Feb;156(2): 378-384. DOI: 10.1177/0194599816683378.

20. Kent DT, Lee JJ, Strollo PJ Jr, et al. Upper airway stimulation for OSA: early adherence and outcome results of one center. Otolaryngol Head Neck Surg 2016;155:188-193. DOI: 10.1177/019459981663 6619.

21. Baptista P, Garaycochea O, Álvarez-Gómez L, et al. Hypoglossal nerve stimulation surgery for obstructive sleep apnoea: our preliminary experience. Acta Otorrinolaringol Esp 2018 Jan-Feb;69(1):42-47. DOI: 10.1016/j.otoeng.2017.12.005.

22. Steffen A, Sommer JU, Hofbauer B, et al. Outcome after one year of upper airway stimulation for obstructive sleep apnea in a multicenter German post-market study. Laryngoscope 2018;128(2):509-515. DOI: 10.1002/lary.26688.

23. Boon M, Huntley C, Steffen A, et al. Upper Airway Stimulation for Obstructive Sleep Apnea: Results from the ADHERE Registry. Otolaryngol Head Neck Surg 2018 Mar 1;159(2):379-385. DOI: 10.1177/0194599818764896..

24. Shah J, Russell JO, Waters T, et al. Uvulopalatopharyngoplasty vs CN XII stimulation for treatment of obstructive sleep apnea: a single institution experience. Am J Otolaryngol 2018 Mar 2;39(3):266-270. DOI: 10.1016/j.amjoto.2018.03.003.

25. Pietzsch JB, Liu S, Garner AM, et al. Long-term cost-effectiveness of upper airway stimulation for the treatment of obstructive sleep apnea: a model-based projection based on the STAR Trial. Sleep 2015;38:735-744. DOI: 10.5665/sleep.4666.
26. Thaler ER, Schwab RJ. Single-institution experience and learning curve with upper airway stimulation. Laryngoscope 2016;126(Suppl 7): S17-S19. DOI: 10.1002/lary.26178.

27. Murphey AW, Baker AB, Soose RJ, et al. Upper airway stimulation for obstructive sleep apnea: the surgical learning curve. Laryngoscope 2016;126:501-506. DOI: 10.1002/lary.25537.

28. Maurer JT, de Vries N. Upper airway stimulation. Implanted neurostimulation device for treatment of obstructive sleep apnea. In Advanced Surgical Techniques in Snoring and Sleep Apnea, Pang KP. ed.; Plural Publishing Inc.; 2013.

29. Maurer JT, De Vries N. Upper airway stimulation for obstructive sleep apnea. Op Tech Otolaryngol Head Neck Surg 2015;26:216-220. DOI: 10.1016/j.otot.2015.10.003.

30. Heiser C, Hofauer B, Lozier L, et al. Nerve monitoring-guided selective hypoglossal nerve stimulation in obstructive sleep apnea patients. The Laryngoscope 2016;126:2852-2858. DOI: 10.1002/lary.26026.

31. Heiser C, Thaler E, Soose R, et al. Technical tips during implantation of selective upper airway stimulation. Laryngoscope 2018 Mar;128(3):756-762. DOI: 10.1002/lary.26724.

32. Steffen A, Kilic A, König IR, et al. Tongue motion variability with changes of upper airway stimulation electrode configuration and effects on treatment outcomes. Laryngoscope 2017 Dec 27;128(8):1970-1976. DOI: 10.1002/lary.27064.

33. Heiser C, Maurer JT, Steffen A. Functional outcome of tongue motions with selective hypoglossal nerve stimulation in patients with obstructive sleep apnea. Sleep Breath 2016;20:553-560. DOI: 10.1007/s11325-015-1237-4.

34. Heiser C. Advanced titration to treat a floppy epiglottis in selective upper airway stimulation. Laryngoscope 2016;126(suppl 7):S22-S24. DOI: $10.1002 /$ lary.26118.

35. Lehman CD, Lee JM, DeMartini WB, et al. Screening MRI in Women With a Personal History of Breast Cancer. J Natl Cancer Inst 2016 Jan 7;108(3):djv349. DOI: 10.1093/jnci/djv349.

36. Mu L, Sanders I. Human tongue neuroanatomy: nerve supply and motor endplates. Clin Anat 2010;23(7):777-791. DOI: 10.1002/ca.21011.

37. Kubin $L$, Jordan AS, Nicholas $C L$, et al. Crossed motor innervation of the base of human tongue. J Neurophysiol 2015;113:3499-3510. DOI: 10.1152/jn.00051.2015.

38. Safiruddin F, Vanderveken OM, de Vries N, et al. Effect of upper-airway stimulation for obstructive sleep apnoea on airway dimensions. Eur Respir J 2015;45(1):129-138. DOI: 10.1183/09031936.00059414.

39. Heiser C, Edenharter G, Bas M, et al. Palatoglossus coupling in selective upper airway stimulation. Laryngoscope 2017 Oct;127(10):E378-E383. DOI: 10.1002/lary.26487.

40. Diercks GR, Wentland C, Keamy D, et al. Hypoglossal Nerve Stimulation in Adolescents With Down Syndrome and Obstructive Sleep Apnea. JAMA Otolaryngol Head Neck Surg 2017 Nov 2;144(1):37-42. DOI: 10.1001/jamaoto.2017.1871. 